\title{
The early-type close binary CV Velorum revisited (Corrigendum)
}

\author{
K. Yakut ${ }^{1,2}$, C. Aerts ${ }^{1,3}$, and T. Morel ${ }^{1}$ \\ ${ }^{1}$ Instituut voor Sterrenkunde, KU Leuven, Celestijnenlaan 200 D, 3001 Leuven, Belgium \\ e-mail: kadriyakut@gmail.com \\ 2 Department of Astronomy and Space Sciences, Ege University, 35100 Izmir, Turkey \\ 3 Department of Astrophysics, Radboud University Nijmegen, PO Box 9010, 6500 GL Nijmegen, The Netherlands
}

A\&A 467, 647-655 (2007), DOI: 10.1051/0004-6361:20065506

Key words. binaries: eclipsing - stars: fundamental parameters - stars: early-type - stars: evolution - stars: oscillations errata, addenda

Some misprints have been identified in the original paper (Yakut et al. 2007). It concerns the labels in Figs. 1 and 5 and some of the wording in Sect. 6. The following corrections should be applied:

- the labels "P" and "S" in Fig. 1 should be interchanged;

- the labels "Primary" and "Secondary" in Fig. 5 should be interchanged;

- in the third paragraph of Sect. 6, " $v \sin i=19 \pm 1 \mathrm{~km} \mathrm{~s}^{-1}$ for the primary and $v \sin i=31 \pm 2 \mathrm{~km} \mathrm{~s}^{-1}$ for the secondary" should read " $v \sin i=19 \pm 1 \mathrm{kms}^{-1}$ for the secondary and $v \sin i=31 \pm 2 \mathrm{~km} \mathrm{~s}^{-1}$ for the primary".
All other reported results and the labelling of the binary components in the other sections of the paper are correct.

Acknowledgements. The authors acknowledge Dr. Simon Albrecht for having pointed out these inconsistencies in our text to us.

\section{References}

Yakut, K., Aerts, C., \& Morel, T. 2007, A\&A, 467, 647 